\title{
THE
}

UNIVERSITY

University of Rhode Island

OF RHODE ISLAND

DigitalCommons@URI

$3-2000$

\section{Numerical Investigation of Boundary Conditions for Moving Contact Line Problems}

Sandesh Somalinga

Arijit Bose

University of Rhode Island, bosea@uri.edu

Follow this and additional works at: https://digitalcommons.uri.edu/che_facpubs

Part of the Chemical Engineering Commons

Terms of Use

All rights reserved under copyright.

\section{Citation/Publisher Attribution}

Somalinga, S., \& Bose, A. (2000). Numerical Investigation of Boundary Conditions for Moving Contact Line Problems. Physics of Fluids, 12(3), 499-510. doi: 10.1063/1.870256

Available at: http://dx.doi.org/10.1063/1.870256

This Article is brought to you for free and open access by the Chemical Engineering at DigitalCommons@URI. It has been accepted for inclusion in Chemical Engineering Faculty Publications by an authorized administrator of DigitalCommons@URI. For more information, please contact digitalcommons-group@uri.edu. 


\title{
Numerical investigation of boundary conditions for moving contact line problems
}

\author{
Sandesh Somalinga and Arijit Bose ${ }^{\text {a) }}$ \\ Department of Chemical Engineering, University of Rhode Island, Kingston, Rhode Island 02881
}

(Received 5 June 1998; accepted 7 October 1999)

\begin{abstract}
When boundary conditions arising from the usual hydrodynamic assumptions are applied, analyses of dynamic wetting processes lead to a well-known nonintegrable stress singularity at the dynamic contact line, necessitating new ways to model this problem. In this paper, numerical simulations for a set of representative problems are used to explore the possibility of providing material boundary conditions for predictive models of inertialess moving contact line processes. The calculations reveal that up to Capillary number $\mathrm{Ca}=0.15$, the velocity along an arc of radius $10 L_{i}$ ( $L_{i}$ is an inner, microscopic length scale) from the dynamic contact line is independent of the macroscopic length scale $a$ for $a>10^{3} L_{i}$, and compares well to the leading order analytical "modulated-wedge" flow field [R. G. Cox, J. Fluid Mech. 168, 169 (1986)] for Capillary number $\mathrm{Ca}<0.1$. Systematic deviations between the numerical and analytical velocity field occur for $0.1<\mathrm{Ca}<0.15$, caused by the inadequacy of the leading order analytical solution over this range of $\mathrm{Ca}$. Meniscus shapes produced from calculations in a truncated domain, where the modulated-wedge velocity field [R. G. Cox, J. Fluid Mech. 168, 169 (1986)] is used as a boundary condition along an arc of radius $R$ $=10^{-2} a$ from the dynamic contact line, agree well with those using two inner slip models for $\mathrm{Ca}<0.1$, with a breakdown at higher $\mathrm{Ca}$. Computations in a cylindrical geometry reveal the role of azimuthal curvature effects on velocity profiles in the vicinity of dynamic contact lines. These calculations show that over an appropriate range of $\mathrm{Ca}$, the velocity field and the meniscus slope in a geometry-independent region can potentially serve as material boundary conditions for models of processes containing dynamic contact lines. (C) 2000 American Institute of Physics.
\end{abstract}

[S1070-6631(00)00402-5]

\section{INTRODUCTION}

Dynamic wetting phenomena, where one liquid spreads across a solid surface while displacing a second immiscible fluid, are ubiquitous in both nature and in industrial processes. Some examples include spreading of insecticides on plants, coating of photographic film, movement of water through interstitial spaces within filter paper fibers, movement of rain drops on windows, cavitation, and flotation of ores during recovery of metals. Common to all of these is the presence of a moving contact line, at the three-phase intersection, and a liquid-fluid boundary extending from this point. The shape of this surface has an important impact on the behavior of these processes. For a given set of materials, moving contact lines can be present in processes that have a range of different macroscopic geometries.

A geometry-independent set of boundary conditions for models of dynamic wetting processes are crucial for a robust evaluation of all the field variables and free-surface shapes, but this produces critical challenges. For a pure liquid, the customary hydrodynamic assumptions lead to a multivalued velocity field at the dynamic contact line. ${ }^{2}$ If the liquid is Newtonian and the solid is nondeformable, this implies an infinite force exerted by the liquid on the solid. Besides its

\footnotetext{
a) Author to whom correspondence should be addressed. Telephone: (401)874-2804; Fax: (401)874-4689; Electronic mail: bosea@egr.uri.edu
}

aphysical nature, one consequence of this force divergence is an unbounded interface curvature at the dynamic contact line, resulting in the inability to specify a "true" dynamic contact angle. Because this angle serves as a boundary condition for the differential equation governing the shape of the liquid-fluid interface, an ill-posed problem results. The lack of a material boundary condition for the differential equation governing the interface shape represents a key obstacle to development of predictive models of dynamic wetting processes. This limitation cannot be overcome by replacing the true dynamic contact angle by an "apparent" one, based upon extrapolation of a static-like meniscus to a putative contact line, because these apparent contact angles depend upon macroscopic geometry. ${ }^{2,3}$ Thus, measurements made in one configuration cannot, in principle, be used in another, severely restricting the usefulness of this approach.

The general strategy for overcoming this problem can be understood by considering the forces that dominate over three different length scales that characterize the flow. In the outer region, characterized by a "macroscopic" length scale " $a$ " (the largest length over which surface tension forces are important), all the usual hydrodynamic assumptions are applicable. For $\mathrm{Ca} \ll 1$ (the Capillary number $\mathrm{Ca}=\mu U / \gamma$, where $U$ is a characteristic liquid speed, $\mu$ the liquid viscosity and $\gamma$ its surface tension), viscous stresses in the outer region are small compared to surface tension forces, the interface shape is static-like to lowest order in $\mathrm{Ca}$ and strongly 
dependent on geometry. As the dynamic contact line is approached, viscous forces grow, and the meniscus shape in this intermediate region is the result of a balance of local viscous forces and surface tension. Because of the local nature of the flow in this region, the interface shape is independent of macroscopic geometry. Within an "inner" region in the vicinity of the dynamic contact line, of characteristic dimensions $L_{i}$, classical hydrodynamics breaks down, and new physics governs fluid behavior. Most common is the assumption of "slip" between the liquid and solid, thereby removing the source of the multivalued velocity at the dynamic contact line; ${ }^{4}$ other postulates include evaporation and condensation at the contact line, ${ }^{5}$ surface diffusion ${ }^{6}$ and modified rheology. ${ }^{7}$ To date, no direct identification of any inner scale mechanism has been made; therefore, its nature remains a matter of speculation. Indirect experiments indicate that the inner scale physics is quite complex, and may arise from multiple mechanisms. ${ }^{8}$

For the double limit $\mathrm{Ca} \rightarrow 0, \epsilon \rightarrow 0$, with $\mathrm{Ca} \ln \left(\epsilon^{-1}\right)$ of $O(1)$, where $\epsilon=L_{i} / a$, singular perturbation analysis ${ }^{1}$ of the resulting boundary value problem to lowest order in $\mathrm{Ca}$, assuming that the displaced fluid has zero viscosity, yields a solution for the interface shape (all primed variables are dimensional) in the intermediate region of the form

$$
\theta_{\text {intermediate }}\left(r^{\prime}\right)=g^{-1}\left[g\left(\theta_{R}\right)+\mathrm{Ca} \ln \left(r^{\prime} / R\right)\right],
$$

where

$$
g(\theta)=\int_{0}^{\theta} \frac{\phi-\operatorname{Cos} \phi \operatorname{Sin} \phi}{2 \operatorname{Sin} \phi} d \phi .
$$

$\theta_{\text {intermediate }}\left(r^{\prime}\right)$ is the angle between the moving solid and the tangent to the liquid free surface (the meniscus slope) in the intermediate region at a distance $r^{\prime}$ from the dynamic contact line, and $\theta_{R}=\theta\left(r^{\prime}=R\right)$, where $r^{\prime}=R$ is a location within the intermediate region. The geometry-free nature of Eq. (1), that is, its lack of dependence on the outer length scale $a$, suggests that it can potentially form a material boundary condition for the outer problem. To be consistent with the dominant role of local viscous forces, the velocity field within this intermediate region must also be invariant with macroscopic geometry. Eliminating the region around the dynamic contact line defined by $r^{\prime}<R$, applying geometry-free boundary conditions for $\theta(R)$ and the flow field on $r^{\prime}=R$, along with the usual boundary conditions at all other interfaces, would permit solution for all the field variables and the free-surface shape in the outer region without specifying any of the details of the fluid physics in the inner region. Since measurements made at an outer length scale cannot be used to uniquely delineate inner scale physics $^{9}$ and because of inherent difficulties associated with probing inner length scale physics experimentally, this approach would represent a significant advance in our ability to predict key field variables in processes containing moving contact lines. This general idea has been explored recently in experiments ${ }^{10-15}$ and in simulations. ${ }^{15-22}$

In this paper, numerical simulations are used as a convenient and powerful probing tool to examine the feasibility of applying the velocity and interface shape in the interme- diate region as transferable (between different macroscopic geometries) boundary conditions for models of processes containing dynamic contact lines. Simulations provide the ability to independently change boundary conditions (or keep them fixed) and controlling parameters in ways that are not possible in experiments. Because calculated meniscus shapes are not subject to "experimental" errors, interface slopes accurate to a specified tolerance that is well beyond currently available experimental techniques can be obtained over the whole computational domain, including, when necessary, the immediate vicinity of the dynamic contact line. This feature allows a refined evaluation of the geometry-independence of any field variables or the meniscus shape. No attempt is made to identify inner scale physics. However, if inner models are available, either through analyses or experiments, in the form mathematical relationships or discrete data, they can be incorporated easily into the calculations.

The Galerkin finite element method is used to solve for interface shapes and flow fields for a set of three model problems. The first consists of a flat plate entering a bath of liquid in a deep rectangular cavity. We use two different inner models and examine the flow field at a specified distance from the dynamic contact line over a range of $\mathrm{Ca}$ and outer length scales. We compare our solutions to those from Cox (referred to as the modulated-wedge velocity field), ${ }^{1}$ in order to determine the limits of applicability for the analytical solution, and identify the parameter space over which that solution can be used as a boundary condition. For the second problem, the computational domain is truncated by an arc of radius $R$ from the dynamic contact line, with $L_{i} \ll R \ll a$, essentially eliminating the inner region from the simulation. Modeling in this domain requires the specification of velocity inflow-outflow boundary conditions along the arc, and we examine the consequences of those conditions on meniscus shapes. In the third problem, a rod enters concentrically into a liquid in a deep cylindrical cavity. This axisymmetric configuration introduces an additional macroscopic geometric parameter, $\delta$, defined as the ratio of the rod radius to the annular gap width. We identify conditions under which this geometric parameter starts playing a measurable role on the velocity field at a specified distance from the dynamic contact line, providing an example of an additional constraint on the applicability of the modulated-wedge flow solution ${ }^{1}$ as a potential material boundary condition.

\section{PROBLEM FORMULATION}

\section{A. Model 1}

The model, shown in Fig. 1, consists of a rectangular cavity of infinite depth, and length, $a$, containing a pure, incompressible Newtonian liquid. The left sidewall moves into the liquid with a constant velocity, $\mathbf{U}$. Gravity is ignored in the simulations, so that the outer length scale becomes the container length, $a$. The upper boundary of the liquid, $h^{\prime}\left(x^{\prime}\right)$, is a free surface whose location is not known a priori, but is obtained as a part of the solution to the problem. A hypothetical lower boundary restricts the computational domain. At this boundary, the flow is assumed unidirectional, with no net volumetric flow, and is sufficiently well removed 


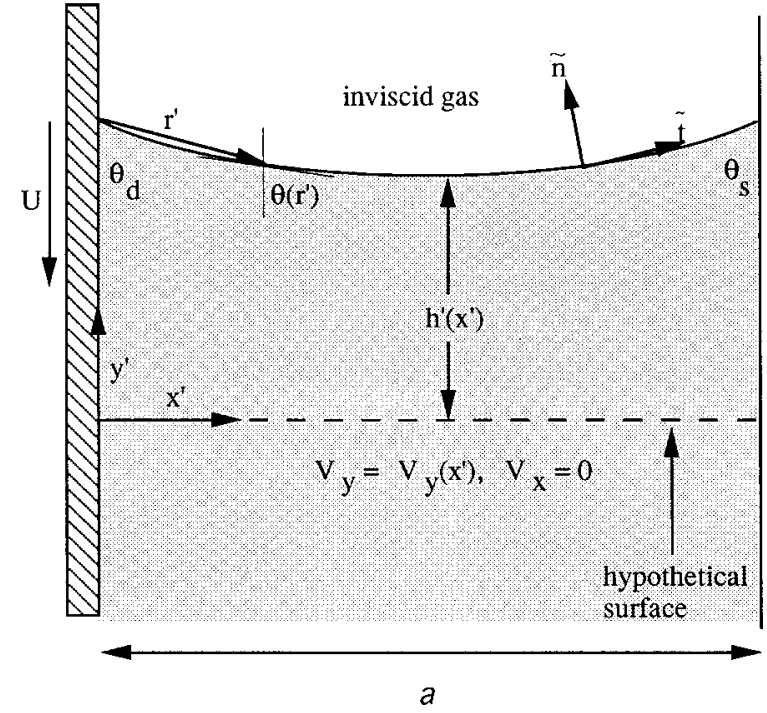

FIG. 1. The model system. A flat plate enters into an infinitely deep bath of liquid at speed $U$. The distance from the moving plate to the stationary side wall is $a$. The dynamic contact angle is $\theta_{D}$, the static contact angle is $\theta_{S}$. A hypothetical lower boundary, across which there is no net mass flux, restricts the computational domain. The flow at this boundary is unidirectional, and it is placed far enough away from the free surface so that its location has no impact on the meniscus shape.

from the free surface so that any further displacement does not have any impact on the shape of the free boundary. The volume per unit width of the liquid within this computational domain is $A$. The static contact angle is $\theta_{S}$. A constant dynamic contact angle $\theta_{D}$ (not necessarily equal to the static contact angle) is used for these simulations. The code can easily handle more complicated variations.

For a single component Newtonian liquid, the Reynolds number, $\mathrm{Re}$, and the Capillary number, $\mathrm{Ca}$, are the dimensionless groups characterizing the problem. Inertial effects have been ignored in the simulations presented here, so that $\mathrm{Re}=0$. The full set of governing equations and boundary conditions are presented in Appendix A. (Note: The influence of inertia on dynamic contact angles has been explored recently $^{23}$ for cases where $\operatorname{Re} \epsilon \ll 1$ with $\operatorname{Re} \gg 1$, and $\operatorname{Re} \epsilon \gg 1$, where the outer length scale is used in the definition of Re.)

\section{B. Model 2}

The truncated computational domain is illustrated in Fig. 2. We apply the slope of the meniscus $\theta\left(r^{\prime}=R\right)=\theta_{R}$ along with a variety of velocity conditions along the arc $r^{\prime}=R$ from the dynamic contact line for this set of calculations. We assume no-slip along all solid-liquid interfaces, different from the slip models utilized in Model 1 for the liquid velocity along moving-solid-liquid interface.

\section{Model 3}

For this set of simulations, illustrated in Fig. 3, a rod of radius $R_{1}$ enters concentrically into liquid in an infinitely deep cylindrical cavity of radius $R_{2}$. We use the exponential slip inner model, and compare the velocity field at $R=10 L_{i}$ with the modulated-wedge flow solution ${ }^{1}$ for different values

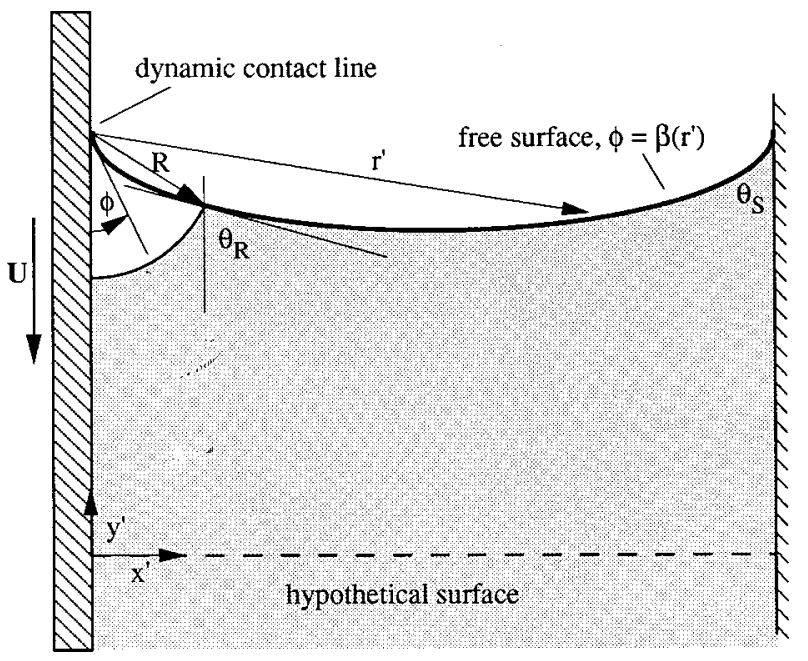

FIG. 2. Illustration of the problem domain remaining after a region $r^{\prime}=R$ from the dynamic contact line is removed. Here $L_{i} \ll R \ll a$. The free surface shape is defined by $\phi=\beta\left(r^{\prime}\right)$.

of $\delta=R_{1} /\left(R_{2}-R_{1}\right)$. The discretization of the flow domain follows the techniques outlined in Bornside ${ }^{24}$ for axisymmetric coating flows.

\section{NUMERICAL SOLUTION TECHNIQUE}

The Galerkin Finite Element technique is used to discretize the continuity and conservation of linear momentum equations, as well as all the boundary conditions. The kinematic condition is used as a distinguished boundary condition to determine the location of the interface. The details leading to the appropriate weak forms of each of these equations follow a standard procedure detailed elsewhere. ${ }^{25}$

The liquid domain is subdivided into $N_{x} \times N_{y}$ elements. Vertical spines originating at the lower boundary of the com-

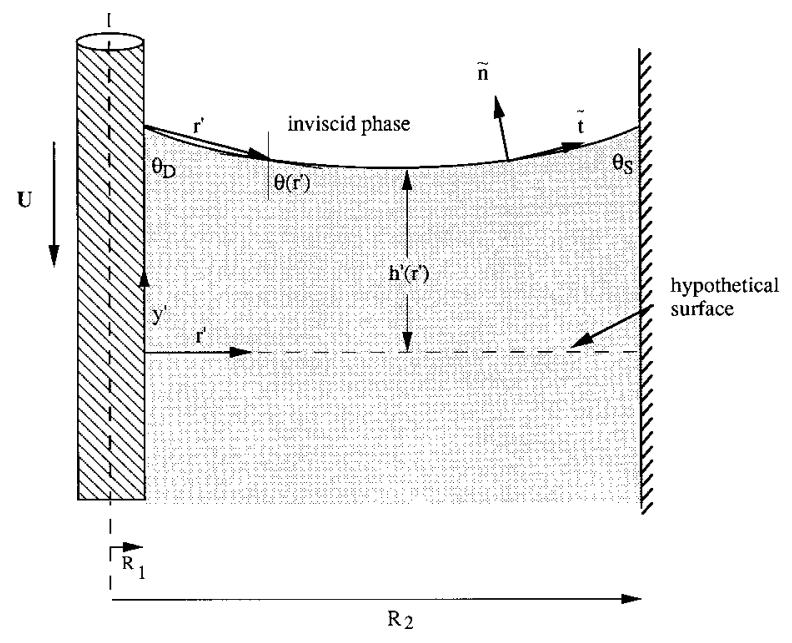

FIG. 3. The model problem for the axisymmetric configuration. A rod of radius $R_{1}$ enters concentrically into a liquid in a container of radius $R_{2}$ at a velocity $U$. The upper boundary is a free surface $h^{\prime}\left(r^{\prime}\right)$. The right wall is stationary. The dynamic contact angle is $\theta_{D}$, while the static contact angle is $\theta_{S}$. The hypothetical lower boundary restricts the computational domain and is far enough away from the free surface so that its location has no impact on the free surface shape. The flow at this location is unidirectional, with no net mass flux across it. 
TABLE I. Maximum-minimum values of variables and their locations for successively refined meshes. $\mathrm{Ca}=5 \times 10^{-3}, \epsilon=10^{-3}, \theta_{S}=\theta_{D}=45^{\circ}$.

\begin{tabular}{|c|c|c|c|c|c|c|c|c|c|}
\hline \multicolumn{10}{|c|}{ Variable values } \\
\hline Mesh size & \multicolumn{2}{|c|}{$\mathrm{V}_{x \text { minimum }}$} & \multicolumn{2}{|c|}{$\mathbf{V}_{y \text { maximum }}$} & \multicolumn{2}{|c|}{$p_{\text {minimum }}$} & \multicolumn{2}{|c|}{$p_{\text {maximum }}$} & $P_{g}$ \\
\hline $22 \times 22$ & \multicolumn{2}{|c|}{$-4.9402 \times 10^{-1}$} & \multicolumn{2}{|c|}{$3.8826 \times 10^{-1}$} & \multicolumn{2}{|c|}{$-5.9068 \times 10^{3}$} & \multicolumn{2}{|c|}{$2.8115 \times 10^{-3}$} & $2.4266 \times 10^{2}$ \\
\hline $23 \times 23$ & \multicolumn{2}{|c|}{$-4.9493 \times 10^{-1}$} & \multicolumn{2}{|c|}{$3.4580 \times 10^{-1}$} & \multicolumn{2}{|c|}{$-8.2526 \times 10^{3}$} & \multicolumn{2}{|c|}{$2.4589 \times 10^{-3}$} & $2.4183 \times 10^{2}$ \\
\hline $25 \times 25$ & \multicolumn{2}{|c|}{$-4.9574 \times 10^{-1}$} & \multicolumn{2}{|c|}{$3.4382 \times 10^{-1}$} & \multicolumn{2}{|c|}{$-1.3561 \times 10^{4}$} & \multicolumn{2}{|c|}{$2.3645 \times 10^{-3}$} & $2.4109 \times 10^{2}$ \\
\hline $26 \times 26$ & -4.957 & $<10^{-1}$ & 3.4390 & $<10^{-1}$ & \multicolumn{2}{|c|}{$-1.3550 \times 10^{4}$} & \multicolumn{2}{|c|}{$2.3645 \times 10^{-3}$} & $2.4109 \times 10^{2}$ \\
\hline \multicolumn{10}{|c|}{ Location } \\
\hline Mesh size & $x$ & $y$ & $x$ & $y$ & $x$ & $y$ & $x$ & $y$ & \\
\hline $22 \times 22$ & 0.1875 & 3.977 & 0.002 & 4.100 & 0.000 & 4.102 & 0.9375 & 0.000 & \\
\hline $23 \times 23$ & 0.1875 & 3.976 & 0.006 & 4.100 & 0.000 & 4.100 & 0.9375 & 0.000 & \\
\hline $25 \times 25$ & 0.1875 & 3.978 & 0.006 & 4.098 & 0.000 & 4.098 & 0.9375 & 0.000 & \\
\hline $26 \times 26$ & 0.1875 & 3.978 & 0.006 & 4.098 & 0.000 & 4.098 & 0.9375 & 0.000 & \\
\hline
\end{tabular}

putational domain form element borders, while the ends of these spines are used to represent the free surface. Element corner nodes are located as desired along the spines. The velocity fields within each element are approximated by nine-node Lagrangian biquadratic basis functions, while the pressure field is approximated by four-node bilinear basis functions. As is customary in finite element practice, the basis functions are developed on a square parent element in a $(\eta, \xi)$ Cartesian coordinate system. This parent element is transformed onto the deformed quadrilateral element in the real domain through the use of an isoparametric mapping. With this mapping, the free surface coincides with $\xi=+1$ for each element bordering the surface. Isoparametric mapping also facilitates the evaluation of the unit normal and tangent vectors. $^{25,26}$ The residuals are calculated using four-point tensor product Gaussian quadrature.

The discretization results in as many nonlinear algebraic equations as the number of unknowns. This algebraic equation set is solved by Newton's method. The linear equation set to be solved at the nth Newton iteration is

$$
\overline{\bar{J}}_{n} \overline{\operatorname{corr}}_{n}=-\bar{R}_{n} \text {, }
$$

where

$$
\overline{\operatorname{corr}}_{n}=\bar{S}_{n+1}-\bar{S}_{n} .
$$

Here $\overline{\mathbf{R}}_{\mathbf{n}}$ is the vector containing the residuals, the elements of $\overline{\mathbf{S}}_{\mathbf{n}}$ consist of the solutions to the field variables and the Jacobian matrix $\overline{\bar{J}}_{\mathbf{n}}=\partial \overline{\mathbf{R}}_{\mathbf{n}} / \partial \overline{\mathbf{S}}_{\mathbf{n}}$. The elements of $\overline{\mathbf{J}}_{\mathbf{n}}$ are obtained numerically using a forward difference scheme. ${ }^{25,26}$ At each Newton iteration, Eq. (3) is solved by frontal elimination. ${ }^{27}$ The Newton iterations are stopped when the $L_{2}$ and $L_{\infty}$ norms of $\overline{\mathbf{R}}_{\mathbf{n}}$ are below $10^{-6}$. Starting from initial guesses where only the essential boundary conditions are specified, along with a static meniscus shape and zero values for all variables at all other nodes, convergence is typically achieved within ten iterations.

\section{RESULTS AND DISCUSSION}

\section{A. Model 1}

As is customary in numerical simulations of transport problems, we first demonstrate that the numerical solution presented is independent of mesh size. Table I illustrates the location and values of $\mathbf{v}_{x \text { minimum }}, \mathbf{v}_{y \text { maximum }}, p_{\text {minimum }}$, $p_{\text {maximum }}$, and $P_{g}$ for successively refined meshes $(N x \times N y$ $=22 \times 22, \quad 23 \times 23, \quad 25 \times 25$, and $26 \times 26)$. Our previous simulations ${ }^{26}$ have clearly demonstrated that the mesh needs to be most refined in the vicinity of the dynamic contact line. The successively finer grids are generated by dividing the first row and column of elements in half. All the variables, as well as their locations, approach constant values as the mesh is refined. We have used the $26 \times 26$ nonuniform mesh, illustrated in Figs. 4(a) and 4(b), for the computations. Our calculations on a $37 \times 37$ mesh, with refinement at several locations in the computational domain, showed no change in

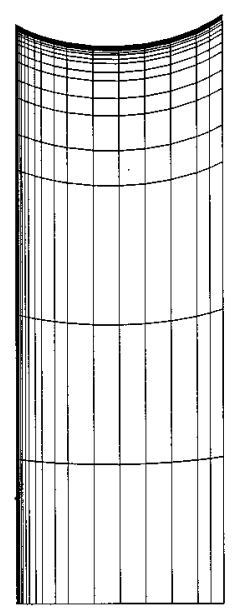

(a)

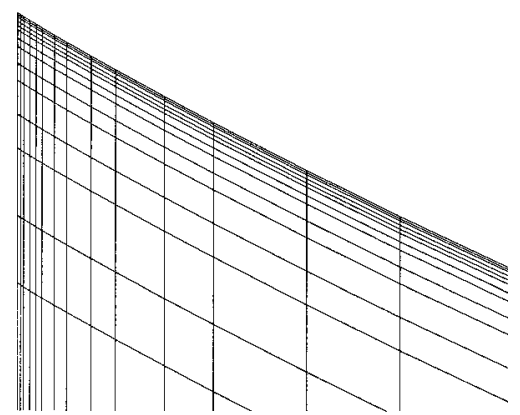

(b)

FIG. 4. (a) The $26 \times 26$ mesh obtained from a converged solution. $\mathrm{Ca}$ $=0.005, \theta_{S}=\theta_{D}=45^{\circ}, \epsilon=10^{-3}$. (b) Expanded view of the mesh in the vicinity of the dynamic contact line. 
either the maximum-minimum values of the variables or their locations from those obtained using the $26 \times 26$ mesh and are thus not reported here.

The lower hypothetical boundary is displaced successively larger distances from the free boundary (through adjustment of the volume/width, $A$ ) until the $L_{2}$ and $L_{\infty}$ norms of the free surface locations from two successive values of $A$ are $<10^{-8}$. Our results indicate that this is achieved when $A=4 a^{2}$, so that this distance is $\sim 4 a$ in these simulations.

Since the meniscus slope at a distance of $r^{\prime}=10 L_{i}$ from the dynamic contact line has been shown previously to be independent of the outer length scale for $\mathrm{Ca}<0.1,{ }^{21}$ we study the flow field at this location to determine conditions over which it is independent of macroscopic geometry. Our simulations permit us to modulate the outer length scale, thus allowing us to examine this issue directly. Figures 5(a)-5(c) are plots of the velocity as a function of angular position along the $\operatorname{arc}(\phi=0$ is the solid). For the range of Ca studied, the velocity field at $r^{\prime}=R=10 L_{i}$ is independent of $a$ for $a$ $>1000 L_{i}$. However, for $a=100 L_{i}$, we find the velocity field is significantly different from that observed at the two larger outer length scales. Since the flow field in the intermediate region must be insensitive to changes in the macroscopic geometry, the location $R=10 L_{i}$ is clearly outside the geometry-free region for this value of $a$. Interpreted in another way, these results show that the velocity at $r^{\prime}$ $>10^{-2} a$ does depend upon macroscopic geometry.

Figures 6(a) and 6(b) are comparisons of both the magnitude and direction of the numerically generated velocity fields along the $\operatorname{arc} R=10 L_{i}\left(a=1000 L_{i}\right.$ in these simulations) to the modulated-wedge solution. ${ }^{1}$ Excellent agreement is maintained up to $\mathrm{Ca}=0.01$. Over this range of $\mathrm{Ca}$, it is apparent that the analytically determined flow field can serve as an appropriate geometry-free boundary condition. However, the agreement begins to deteriorate at $\mathrm{Ca}=0.1$. For the modulated-wedge velocity field to properly describe the flow, the interface curvature in the intermediate region should be small, that is, $(d \beta / d \ln r)^{2} \ll 1$, where the shape of the interface is described by $\phi=\beta(r)$. Figure 7 shows $\left(d \beta / d \ln r^{*}\right)^{2}$ versus $r^{*}$ for a range of $\mathrm{Ca}\left(r^{*}=r^{\prime} / L_{i}\right.$ $=r / \epsilon)$. Even at the highest value of $\mathrm{Ca}$, the maximum value of this quantity is $\sim 0.05$ for $5<r^{*}<40$, so that the low interface slope condition required for the validity of the modulated-wedge solution appears to hold. Thus, the most likely cause for this discrepancy is the inadequacy of the leading order solution at this $\mathrm{Ca}$.

\section{B. Model 2}

A pie-shaped region defined by $r^{\prime}=10 L_{i}$ from the dynamic contact line is removed from consideration. Table II illustrates the magnitudes and locations of maxima and minima in the field variables for successively refined meshes. We have used the $21 \times 21$ nonuniform mesh illustrated in Figures 8(a) and 8(b) for the computations carried out in this truncated domain. While very refined grids in the immediate vicinity of the moving contact line region become necessary in Model 1 to capture the large stresses in the immediate vicinity of the dynamic contact line, elimination of this re-
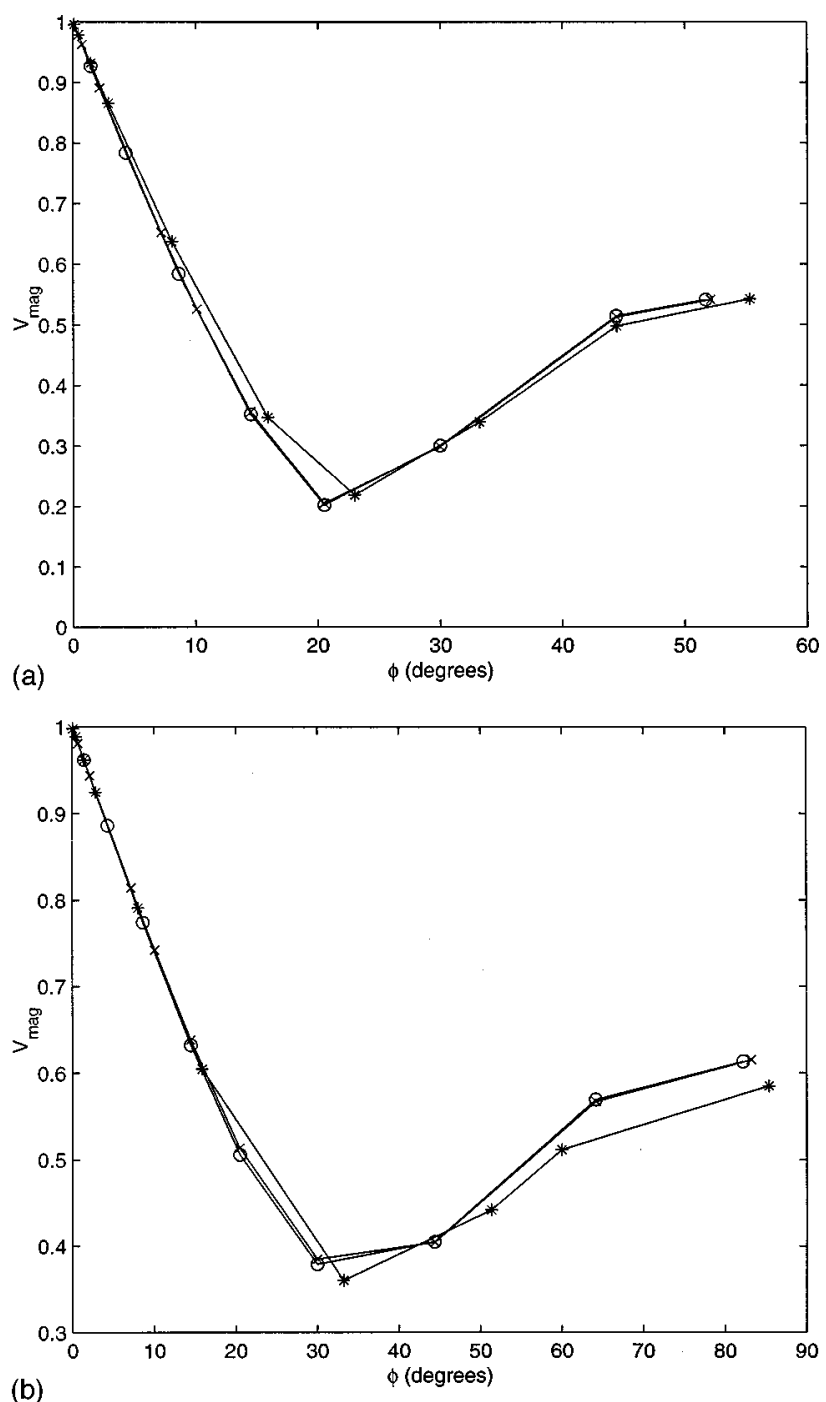

(b)

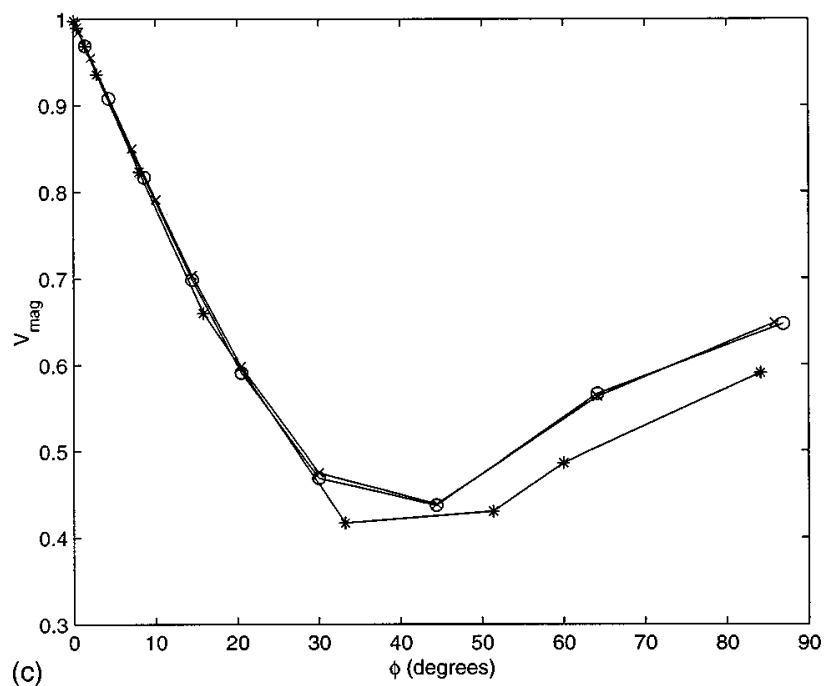

FIG. 5. Magnitude of velocity at $r^{\prime}=10 L_{i}$ vs polar angle $\phi$ for different values of the outer length scale. $\theta_{S}=\theta_{D}=45^{\circ}$. (****) $a=100 L_{i}$; $(++++) a=1000 L_{i} ;(\bigcirc \bigcirc \bigcirc \bigcirc) a=2000 L_{i}$. (a) $\mathrm{Ca}=0.01$, (b) $\mathrm{Ca}=0.1$, (c) $\mathrm{Ca}=0.15$.

gion here allows the use of coarser meshes. Note that with the exception of $p_{\text {minimum }}$, all of the field variables have converged to approximately the same values as those in Model 1 , providing a preliminary indication of the success of this 

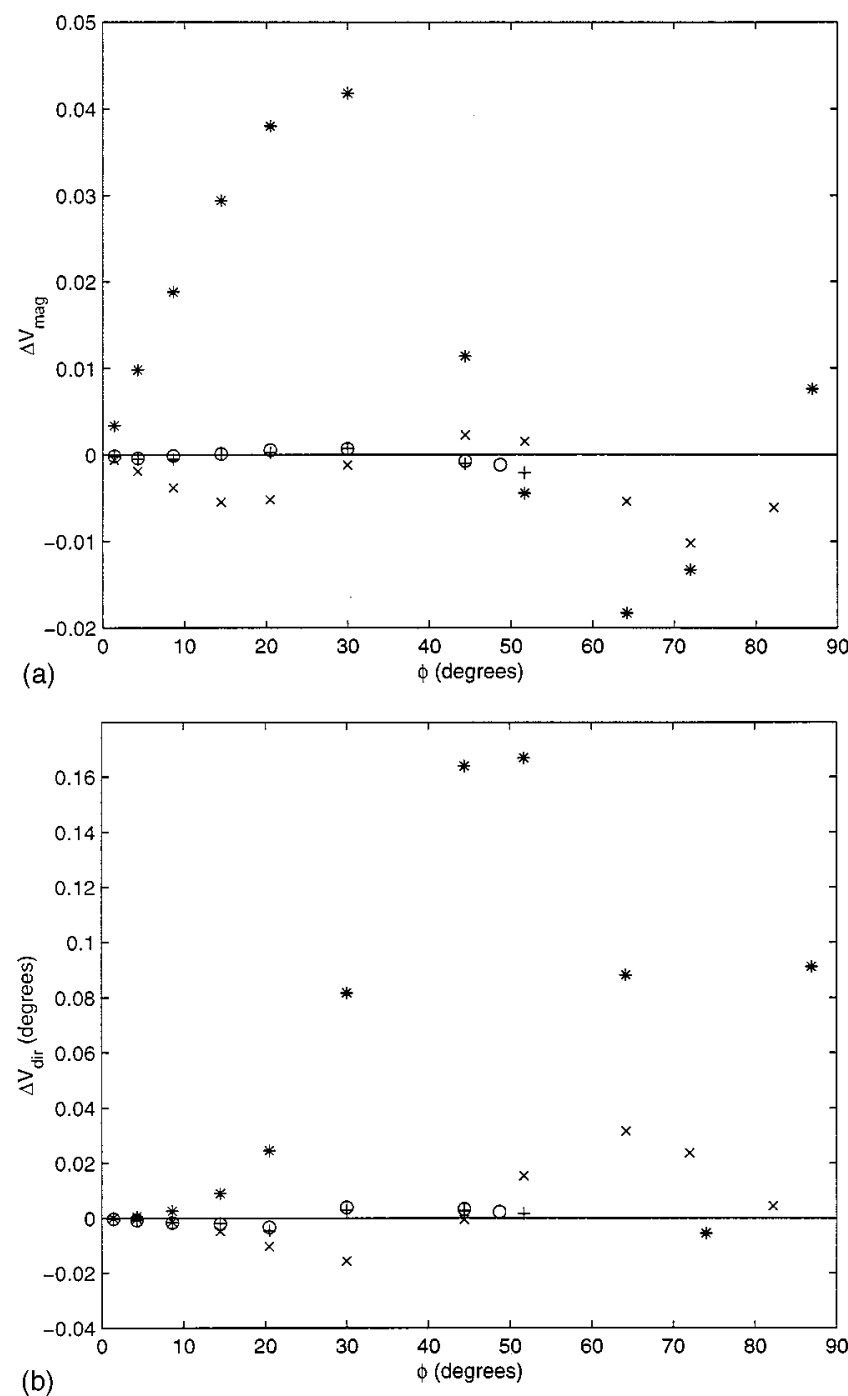

FIG. 6. Difference of the (a) magnitude and (b) direction, of the velocities at $r^{\prime}=10 L_{i}$ between the simulation and the modulated-wedge velocity field. (Ref. 1) $\epsilon=5 \times 10^{-4}, \theta_{S}=\theta_{D}=45^{\circ} .(\bigcirc \bigcirc \bigcirc \bigcirc) \mathrm{Ca}=0.005 ;(++++) \mathrm{Ca}$ $=0.01 ;(\times \times \times \times) \mathrm{Ca}=0.1 ;(* * * *) \mathrm{Ca}=0.15$.

method for modeling dynamic wetting processes. The discrepancy for $p_{\text {minimum }}$ is because this value is achieved at the dynamic contact line for the full domain, a point which is not part of the computational domain in this problem.

The meniscus slope boundary condition $\theta=\theta_{R}$ is applied at the point of intersection of the arc with the free surface. The modulated-wedge ${ }^{1}$ velocity field is applied as an inflow/ outflow condition along the arc $r^{\prime}=R\left(R=10^{-2} a\right)$ drawn from the point of intersection of the tangent to the free surface at $R$ and the moving solid (this location is called the apparent dynamic contact line). The no-slip condition is applied everywhere along the solid-liquid boundaries. The resulting interface slopes are compared to those using the exponential slip inner model in Figures 9(a)-9(d). Interface slopes match well up to $\mathrm{Ca}=0.1$ but discrepancies set in at $\mathrm{Ca}=0.15$. Interface profiles calculated using another inner model shown in Appendix A for $\epsilon=10^{-3}$ (therefore, $r^{\prime}$ also equals $10 L_{i}$ ) were indistinguishable from those using the exponential slip inner model. Thus, for $\mathrm{Ca}<0.1$, two different inner slip models produce free surface shapes that compare

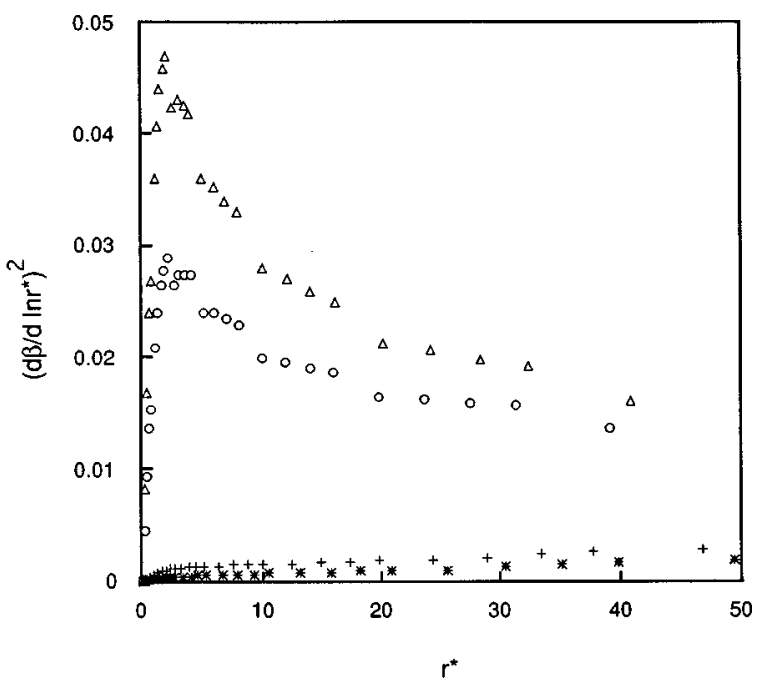

FIG. 7. Interface curvature measure $\left(d \beta / d \ln r^{*}\right)^{2}$ vs distance $r^{*}$ from dynamic contact line $\left(r^{*}=r^{\prime} / L_{i}=r / \epsilon\right) .(* * * *) \mathrm{Ca}=0.005 ;(++++) \mathrm{Ca}$ $=0.01 ;(\bigcirc \bigcirc \bigcirc) \mathrm{Ca}=0.1 ;(\triangle \triangle \triangle \triangle) \mathrm{Ca}=0.15$.

well with that obtained using the modulated-wedge velocity field on the truncated domain defined by $r^{\prime}=10 L_{i}$, providing a strong indication of the validity of this modeling strategy.

The kinematic condition is used as a distinguished boundary condition to determine the location of the free surface. At $r^{\prime}=R$, drawn from the apparent dynamic contact line, the meniscus slope $\theta_{R}$ replaces the kinematic condition in order to avoid overspecifying the problem. Because of local changes in interface curvature, the position of this apparent contact line becomes increasingly different from the true one as $\mathrm{Ca}$ increases. One important consequence of this displacement is illustrated in Table III, which shows the normal component of velocity at the intersection of the arc $r^{\prime}$ $=R$ and the free surface. The kinematic condition is violated, with the magnitude of the normal velocity increasing with increasing $\mathrm{Ca}$. In order to apply the modulated-wedge boundary condition, it is necessary to know the location of the true dynamic contact line. The violation of the kinematic condition in this model is a consequence of the fact that the $\operatorname{arc} r^{\prime}=R$ has been constructed from the apparent dynamic contact line. If Moffat's wedge flow solution ${ }^{28}$ constructed from that same point is used as a boundary condition, ${ }^{22}$ it would satisfy the kinematic condition, but it would not properly account for viscous bending effects in the intermediate region, an effect that becomes more important as $\mathrm{Ca}$ increases. Direct experimental measurements of this velocity field could also be used as an input in these simulations. If the dynamic contact line can be located successfully, this velocity field would be specified at a distance from the contact line equal to some fraction of the outer length scale, and it would satisfy the kinematic condition. The simulations would proceed without any problems. Use of the analytical modulated-wedge flow solution as a boundary condition for the simulations is therefore restricted, and underscores the need for direct experimental measurements.

The unique capacity of numerical simulations is now exploited to determine how sensitive the free boundary 
TABLE II. Maximum-minimum values of variables and their locations for successively refined meshes (Truncated domain). Ca $=5 \times 10^{-3}, R=10^{-2} a$, $\theta_{R}=\theta_{S}=45^{\circ}$.

\begin{tabular}{|c|c|c|c|c|c|c|c|c|c|}
\hline \multicolumn{10}{|c|}{ Variable values } \\
\hline Mesh size & \multicolumn{2}{|c|}{$\mathrm{V}_{x \text { minimum }}$} & \multicolumn{2}{|c|}{$\mathbf{V}_{y}$ maximum } & \multicolumn{2}{|c|}{$P_{\text {minimum }}$} & \multicolumn{2}{|c|}{$P_{\text {maximum }}$} & $P_{g}$ \\
\hline $18 \times 18$ & \multicolumn{2}{|c|}{$-4.9283 \times 10^{-1}$} & \multicolumn{2}{|c|}{$3.4012 \times 10^{-1}$} & \multicolumn{2}{|c|}{$-4.0479 \times 10^{2}$} & \multicolumn{2}{|c|}{$7.2035 \times 10^{-2}$} & $2.4335 \times 10^{2}$ \\
\hline $20 \times 20$ & \multicolumn{2}{|c|}{$-4.9324 \times 10^{-1}$} & \multicolumn{2}{|c|}{$3.4613 \times 10^{-1}$} & \multicolumn{2}{|c|}{$-4.0919 \times 10^{2}$} & \multicolumn{2}{|c|}{$1.3723 \times 10^{-2}$} & $2.4330 \times 10^{2}$ \\
\hline $21 \times 21$ & \multicolumn{2}{|c|}{$-4.9327 \times 10^{-1}$} & \multicolumn{2}{|c|}{$3.4711 \times 10^{-1}$} & \multicolumn{2}{|c|}{$-4.0605 \times 10^{2}$} & \multicolumn{2}{|c|}{$9.8965 \times 10^{-3}$} & $2.4329 \times 10^{2}$ \\
\hline $23 \times 23$ & \multicolumn{2}{|c|}{$-4.9327 \times 10^{-1}$} & \multicolumn{2}{|c|}{$3.4717 \times 10^{-1}$} & \multicolumn{2}{|c|}{$-4.0569 \times 10^{2}$} & \multicolumn{2}{|c|}{$1.0140 \times 10^{-2}$} & $2.4329 \times 10^{2}$ \\
\hline $25 \times 25$ & \multicolumn{2}{|c|}{$-4.9327 \times 10^{-1}$} & \multicolumn{2}{|c|}{$3.4717 \times 10^{-1}$} & \multicolumn{2}{|c|}{$-4.0564 \times 10^{2}$} & \multicolumn{2}{|c|}{$1.0096 \times 10^{-2}$} & $2.4329 \times 10^{2}$ \\
\hline \multicolumn{10}{|c|}{ Location } \\
\hline Mesh size & $x$ & $y$ & $x$ & $y$ & $x$ & $y$ & $x$ & $y$ & \\
\hline $18 \times 18$ & 0.1875 & 3.977 & 0.0076 & 4.100 & 0.0076 & 4.096 & 0.9375 & 0.000 & \\
\hline $20 \times 20$ & 0.1875 & 3.976 & 0.0098 & 4.100 & 0.0076 & 4.096 & 0.9375 & 0.000 & \\
\hline $21 \times 21$ & 0.1875 & 3.978 & 0.0098 & 4.098 & 0.0076 & 4.096 & 0.9375 & 0.000 & \\
\hline $23 \times 23$ & 0.1875 & 3.978 & 0.0098 & 4.098 & 0.0076 & 4.096 & 0.9375 & 0.000 & \\
\hline $25 \times 25$ & 0.1875 & 3.978 & 0.0098 & 4.098 & 0.0076 & 4.096 & 0.9375 & 0.000 & \\
\hline
\end{tabular}

shapes are to different velocities prescribed at $r^{\prime}=R$. Clearly, such a "sensitivity" test can only be performed using computations, since there is no way to deliberately produce prescribed velocities at specific locations within the liquid in experiments. These computations can aid experimentalists in determining the required level of accuracy for velocity measurements in anticipation of their use as boundary conditions. We have applied three new velocity boundary

(a)
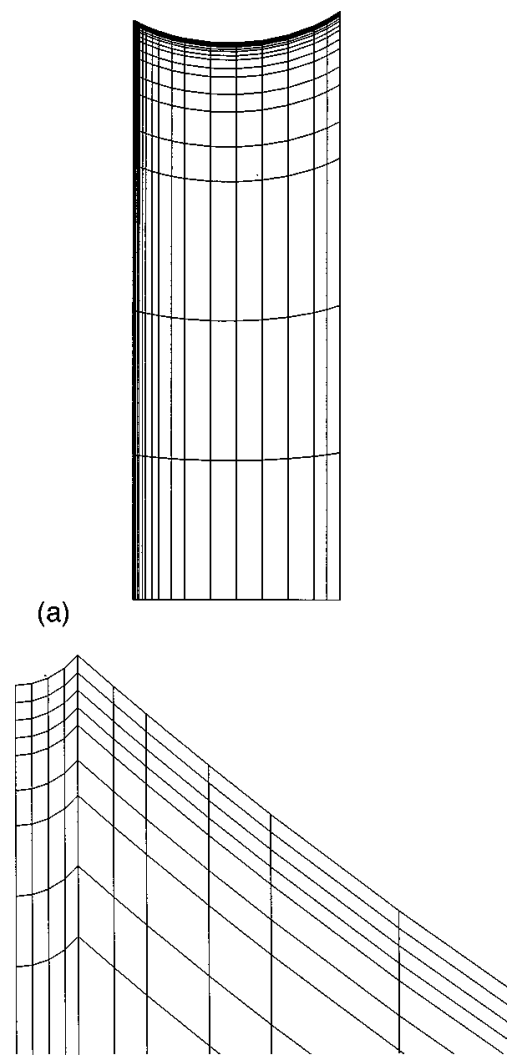

(b)

FIG. 8. (a) The $21 \times 21$ mesh used for simulations in a domain where a region $r^{\prime}=R=10^{-2} a$ from an apparent dynamic contact line is removed from the computational domain. $\mathrm{Ca}=0.005, \theta_{S}=\theta_{D}=45^{\circ}$. (b) An expanded view of the mesh showing the truncated region. conditions [Eqs. (5)-(7)] along the arc $r^{\prime}=R=10^{-2} a$. These have been chosen to satisfy no net mass flux across the $\operatorname{arc} r^{\prime}=R$ and no-slip at the walls, but otherwise lack any physical basis, and are illustrated in Fig. 10.

Type (1) $\quad \mathrm{v}_{\phi}=0, \quad \mathrm{v}_{r}=\cos \left[\pi \times \phi / \theta_{R}\right], \quad 0 \leqslant \phi \leqslant \theta_{R}$,

Type (2) $\quad \mathrm{v}_{\phi}=0, \quad \mathrm{v}_{r}=\cos \left[2 \times \pi \times \phi / \theta_{R}\right], \quad 0 \leqslant \phi \leqslant \theta_{R}$,

Type (3) $\quad \mathrm{v}_{\phi}=0, \quad \mathrm{v}_{r}=1-\left[4 \times \phi / \theta_{R}\right], \quad \phi \leqslant \theta_{R} / 4$,

$$
\begin{aligned}
& \mathrm{v}_{r}=\sin \left[4 \times \pi \times \phi / \theta_{R}\right], \quad \theta_{R} / 4<\phi \leqslant 3 \theta_{R} / 4, \\
& \mathrm{v}_{r}=3-\left[4 \times \phi / \theta_{R}\right], \quad 3 \theta_{R} / 4<\phi \leqslant \theta_{R} .
\end{aligned}
$$

The resulting interface slopes are compared with those obtained using the modulated-wedge velocity boundary condition at $r^{\prime}=R$, for a range of $\mathrm{Ca}$, and are shown in Figs. 11(a)-11(d). For $\mathrm{Ca}<0.1$, the interface profiles produced from each of these new models are indistinguishable from that using the modulated-wedge flow field at the scale of the plots. Therefore, for this $\mathrm{Ca}$ regime and for this resolution, it is not necessary to know the exact form of the velocity boundary condition along the $\operatorname{arc} r^{\prime}=R$ in order to produce "accurate" meniscus shapes. Considering the amount of additional effort that must be expended to gather velocity information, this type of computation can lead to important savings when modeling over a specific parameter range. Significant systematic deviations can, however, be observed at higher $\mathrm{Ca}$, so that for $\mathrm{Ca}>0.1$, precise flow field boundary conditions must be utilized.

\section{Model 3}

As was done and reported for the flat plate geometry, extensive computations are first completed to identify conditions where the solution is independent of mesh size, and the lower hypothetical boundary is located sufficiently far away from the free surface. A $26 \times 26$ mesh, identical to that used in the flat plate geometry proved to be sufficient in these calculations. The outer length scale $a=R_{2}-R_{1}$. The lower 

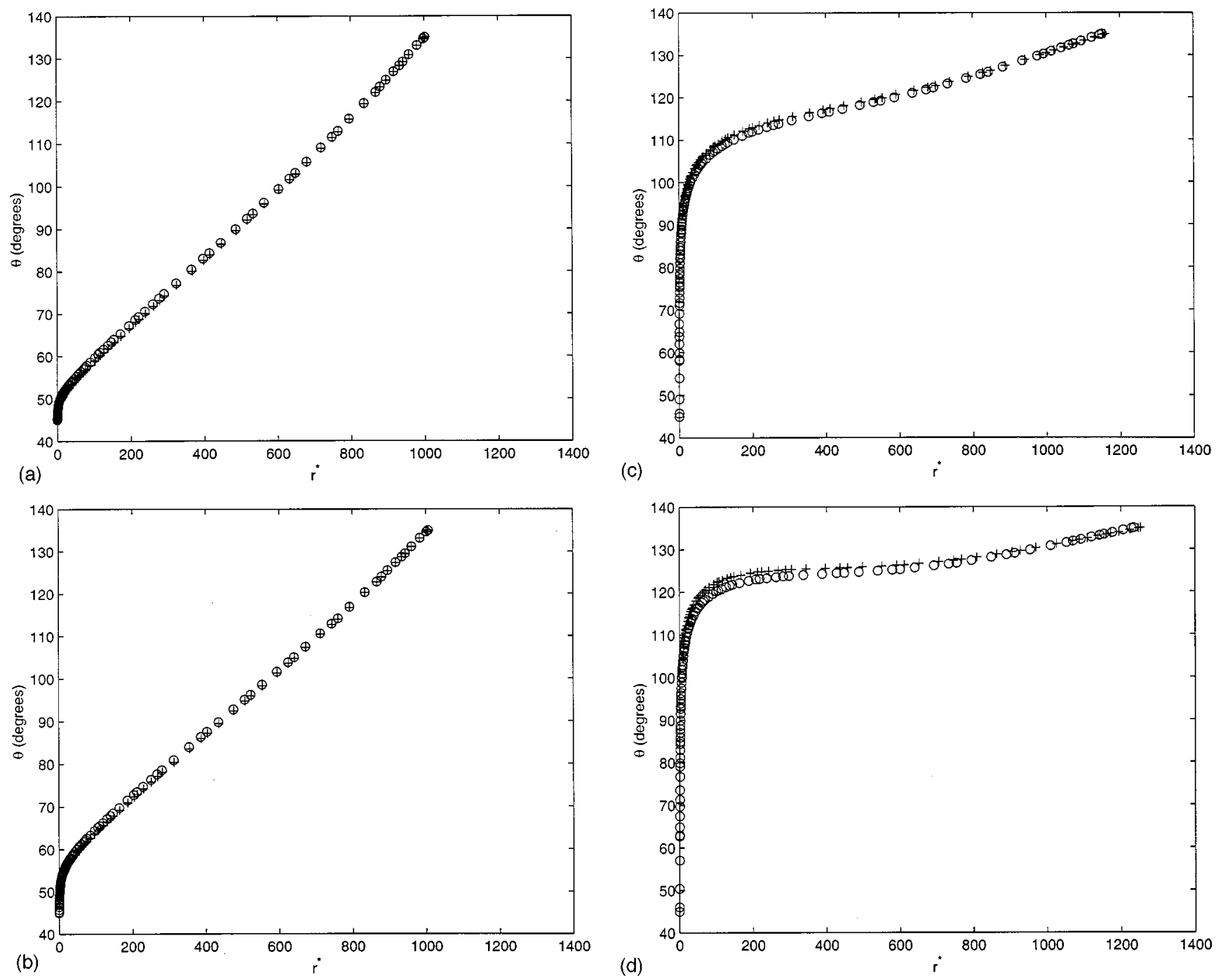

FIG. 9. Comparisons of interface slopes from the simulations using an exponential slip model with $\epsilon=10^{-3}(\bigcirc \bigcirc \bigcirc \bigcirc)$ and simulations using the modulated wedge velocity field (Ref. 1) as a boundary condition along the $\operatorname{arc} r^{\prime}=R=10 L_{i}(++++)$. The interface slope $\theta_{R}$ is the best fit value obtained from our previous simulations. (Ref. 23). The variable $r^{*}=r^{\prime} / L_{i}$. (a) $\mathrm{Ca}=0.005$, (b) $\mathrm{Ca}=0.01$, (c) $\mathrm{Ca}=0.1$, (d) $\mathrm{Ca}=0.15$.

boundary was placed approximately $6\left(R_{2}-R_{1}\right)$ away from the free surface. This distance is adjusted by varying the total volume of liquid in the annular space.

The flow field is axisymmetric and two-dimensional in the $r^{\prime}-y^{\prime}$ plane. The key results are shown in Fig. 12, where the difference in the magnitudes as well as directions of the velocities at $r^{\prime}=R=10 L_{i}$ between the modulatedwedge flow solution ${ }^{1}$ and the numerical calculation for $\mathrm{Ca}$ $=0.005$, are shown for various values of $\delta$. For $\delta>1$, the planar modulated-wedge solution mimics the calculated

TABLE III. Normal component of velocity at the point of intersection of the $\operatorname{arc} r^{\prime}=R=10^{-2} a$ with the free surface.

\begin{tabular}{ll}
\hline \hline $\mathrm{Ca}$ & $\mathbf{n . V}$ \\
\hline 0.005 & 0.011 \\
0.01 & 0.0191 \\
0.1 & 0.0783 \\
0.15 & 0.0846 \\
\hline \hline
\end{tabular}

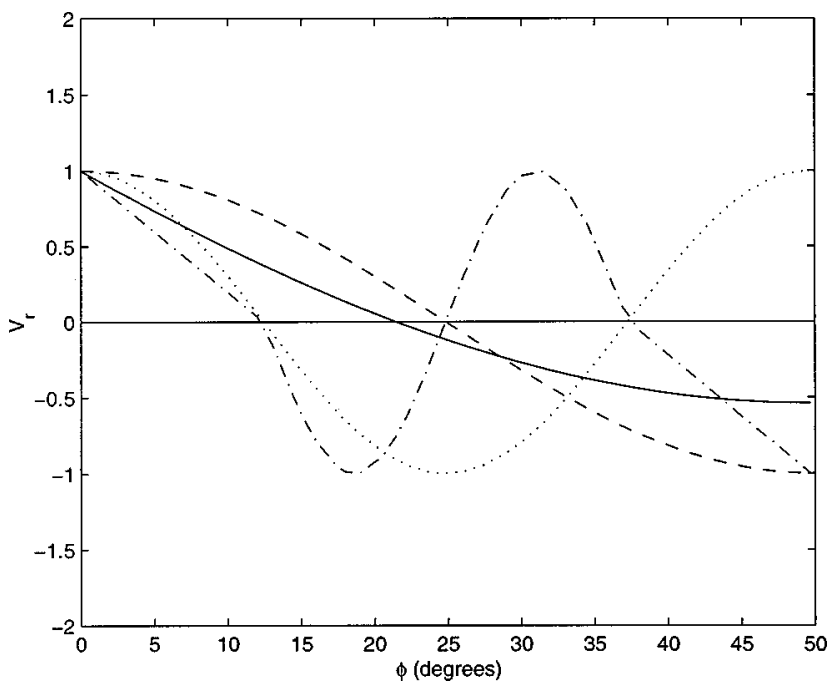

FIG. 10. Plots of the radial component of velocity vs polar angle $\phi$ for the different velocity fields used as boundary conditions at $r^{\prime}=10 L_{i}$. (----) Type $1 ;(\cdots)$ Type $2 ;(-\cdot-\cdot)$ Type $3 ;(-)$ Cox. ${ }^{1}$ 

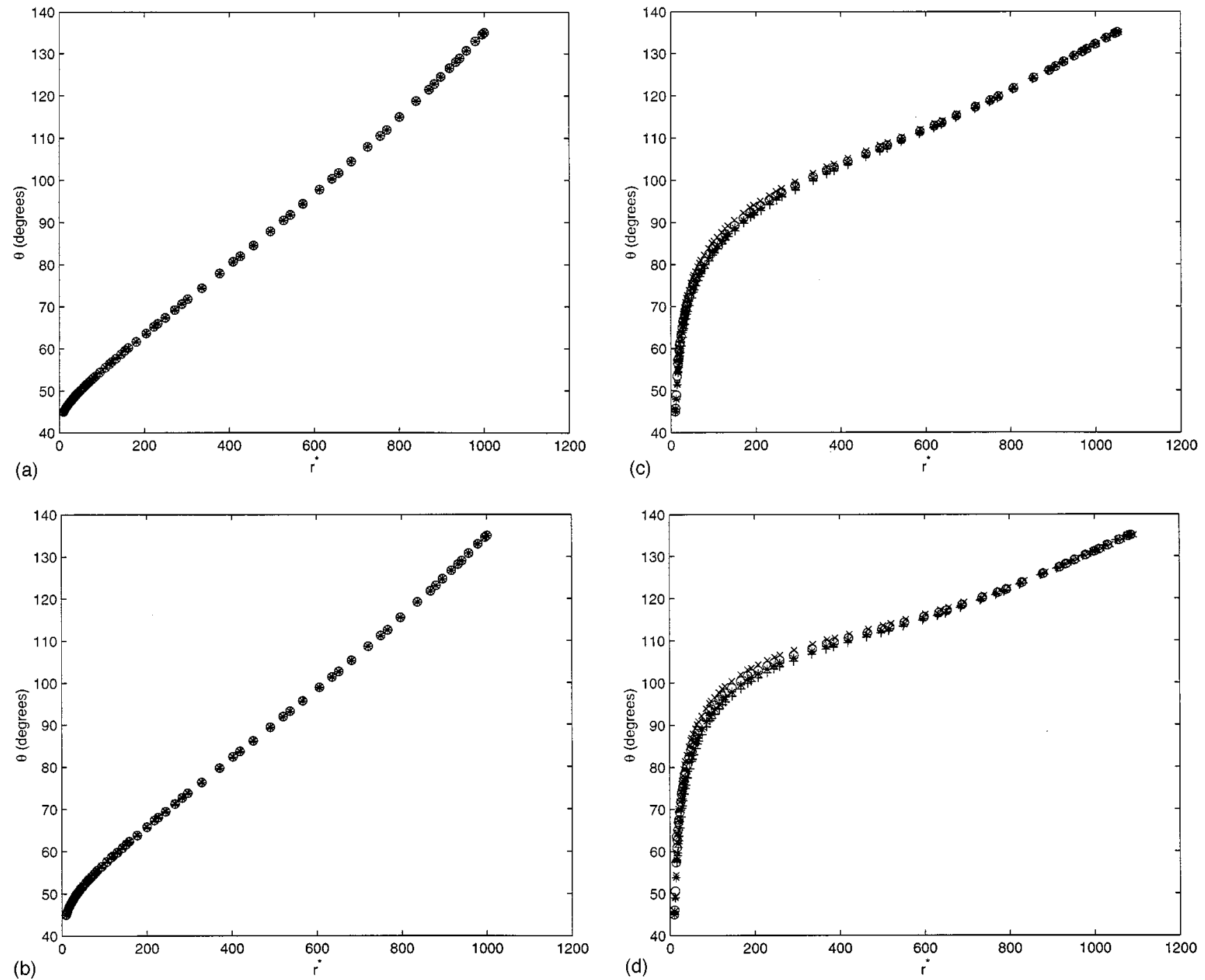

FIG. 11. Comparisons of simulated interface slopes using the three hypothetical velocity boundary conditions with that using the modulated wedge velocity field, all applied at $r^{\prime}=R=10^{-2} a .(++++)$ Type $1 ;(\times \times \times \times)$ Type 2; (****) Type 3; (○○○○) Modulated wedge. (a) $\mathrm{Ca}=0.005,(\mathrm{~b}) \mathrm{Ca}=0.01,(\mathrm{c})$ $\mathrm{Ca}=0.1$, and (d) $\mathrm{Ca}=0.15$.

velocity field. However, the discrepancy becomes very evident at $\delta=0.1$, where the role of inner rod curvature on the flow field is significant. The $\delta$ value at which deviations from the planar modulated-wedge flow field set in are dependent on the choice of $R$ as well as on Ca. This calculation is an example of how simulations can be exploited to put a bound on the use of the modulated-wedge flow velocity field ${ }^{1}$ as a geometry-independent boundary condition.

\section{CONCLUSIONS}

Numerical simulations of a plate being immersed into an infinitely deep liquid bath have been utilized to test a strategy aimed at providing geometry-free boundary conditions for the simulation of dynamic wetting processes. The flow field along an arc of radius $10 L_{i}$ drawn from the dynamic contact line is compared to the prediction from the modulated-wedge solution. The two velocity fields are in good agreement for $\mathrm{Ca}<0.1$. The discrepancy at higher val- ues of $\mathrm{Ca}$ is not a result of a violation of the low curvature assumption, but is most likely because the solution to lowest order in $\mathrm{Ca}$ is inadequate. The problem is modeled by eliminating a region defined by $r^{\prime}=10^{-2} a$ around the dynamic contact line, and applying velocity and interface slope boundary conditions along this line. When the modulatedwedge solution is used as a velocity boundary condition along the arc, the resulting interface shapes were found to be in good agreement with those obtained using the exponential slip inner model for $\mathrm{Ca}<0.1$. Computations completed using a different slip model produced no difference in interface shapes, establishing the viability of using the truncated domain to model wetting processes. Our study indicated a relative insensitivity of the meniscus shape to the nature of the velocity boundary conditions applied on the truncated domain for $\mathrm{Ca}<0.1$. Using computations in an axisymmetric geometry, we identify conditions where the planar modulated-wedge flow velocity field breaks down because of 


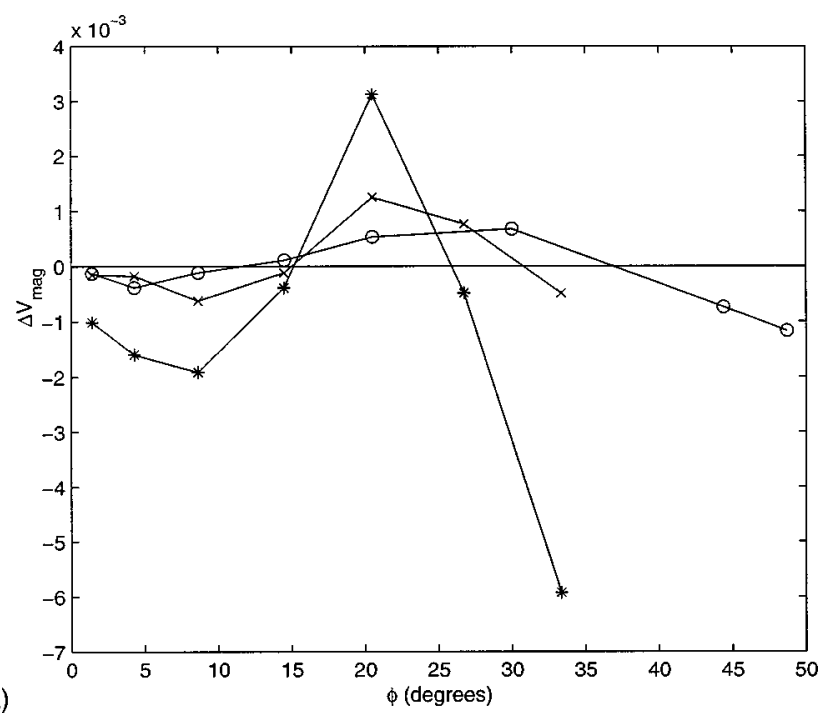

(a)

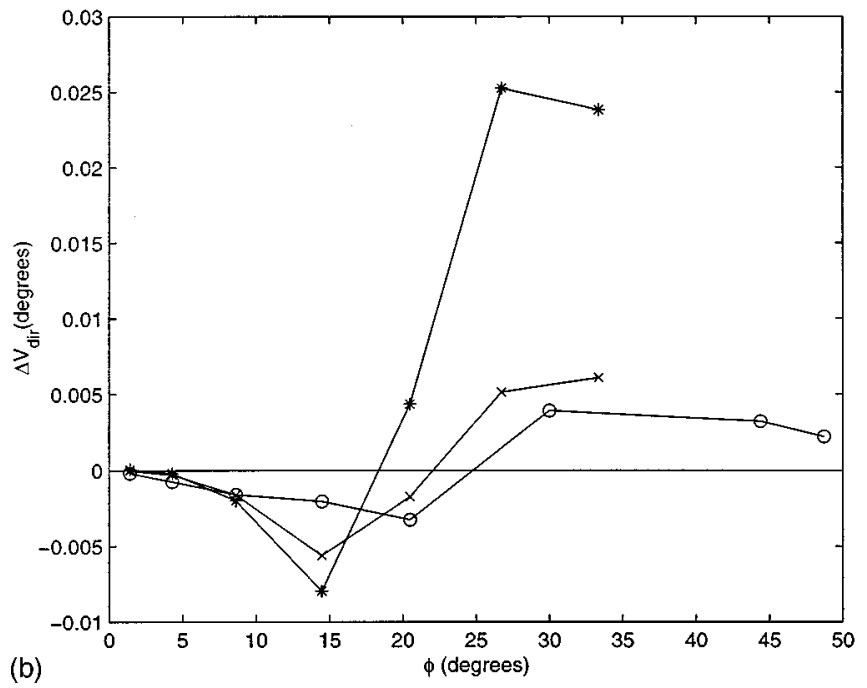

FIG. 12. Comparison of the (a) magnitude and (b) direction, of the velocities at $r^{\prime}=10 L_{i}$ from the simulation in an axisymmetric geometry with the modulated-wedge velocity field (Ref. 1). $\epsilon=5 \times 10^{-4}, \theta_{S}=\theta_{D}=45^{\circ}, \mathrm{Ca}$ $=0.005, \delta=R_{1} /\left(R_{2}-R_{1}\right) .(\bigcirc \bigcirc \bigcirc \bigcirc)$ Flat plate, $\delta \gg 1 ;(\times \times \times \times) \delta=1$; $(* * * *) \delta=0.1$.

azimuthal curvature effects, putting another useful bound on the applicability of the analytical solution as a geometryindependent boundary condition.

\section{ACKNOWLEDGMENTS}

We thank E. Ramé, S. Garoff, and M. Rivero-Hudec for critical insights and extensive discussions. This work was supported in part by the Universities Space Research Association through the National Center for Microgravity Research, and Grant No. NAG-3-2129 from the NASA Lewis Research Center. Computations were carried out at the Pittsburgh Supercomputer Center and the San Diego Supercomputer Center.

\section{APPENDIX A: GOVERNING EQUATIONS AND BOUNDARY CONDITIONS}

\section{Problem 1}

\section{A. Governing equations}

All lengths are scaled with $a$, the container length, velocities with the plate speed, $U$, and pressure and viscous stresses with $\mu U / a$. All variables presented here are dimensionless.

\section{B. Continuity}

$$
\nabla \cdot \mathbf{v}=0 \text {. }
$$

Here $\mathbf{v}(x, y)=\mathbf{v}_{x}(x, y) \mathbf{e}_{\mathbf{x}}+\mathbf{v}_{y}(x, y) \mathbf{e}_{\mathbf{y}}$, where $\mathbf{e}_{\mathbf{x}}$ and $\mathbf{e}_{\mathbf{y}}$ are unit vectors in the $x$ and $y$ directions, respectively.

\section{Conservation of momentum (with $R e=0$ )}

$$
\nabla \cdot \mathbf{T}=0 .
$$

The stress tensor, $\mathbf{T}=-p \mathbf{I}+\tau$, where $\mathbf{I}$ is the identity tensor, and $p$ is the liquid pressure. The viscous stress tensor, assuming a Newtonian rheology, is of the form $\tau=\left[\nabla_{\mathbf{v}}+\left(\nabla_{\mathbf{v}}\right)^{T}\right]$.

\section{Boundary conditions}

No slip and no penetration of liquid on all stationary solid walls

$$
\mathbf{v}=\mathbf{0} \text {. }
$$

At the free surface, $y=h(x)$

$$
\begin{aligned}
& \mathbf{n} \cdot \mathbf{T}=(1 / \mathrm{Ca}) 2 H \mathbf{n}-\mathbf{n} P_{g}, \\
& \mathbf{n} \cdot \mathbf{v}=0 .
\end{aligned}
$$

Equations (A4) and (A5) represent the stress balance and the kinematic conditions, respectively. Here, $\mathbf{n}$ is the unit outward normal from the free liquid surface, $H$ is the mean curvature of the interface, and $P_{g}$ is the unknown pressure in the surrounding inviscid phase, relative to an arbitrary chosen datum pressure at the lower right hand corner of the computational domain.

The contact angles are specified at the contact lines, so that

$$
\mathbf{n}_{\text {solid }} \cdot \mathbf{n}=\cos (\theta) .
$$

At the dynamic contact line, the microscopic dynamic contact angle $\theta=\theta_{D}$ and at the static contact line, $\theta=\theta_{S}$.

Along the moving plate, the liquid speed is given by a slip model. We have investigated two different slip models: Exponential slip:

$$
\mathrm{v}_{y}=-[1-\exp \{-(h(0)-y) / \epsilon\}] .
$$

Another model: ${ }^{9}$

$$
\mathbf{v}_{y}=-\{(h(0)-y) / \epsilon\}^{2} /\left[1+\{(h(0)-y) / \epsilon\}^{2}\right],
$$

where $h(x)$ is the dimensionless height of free surface and $\boldsymbol{\epsilon}=L_{i} / a$. These models directly produce $\mathbf{v}=\mathbf{0}$ at the contact line. The key difference between them is how this limiting velocity is approached.

At the hypothetical lower surface, $y=0$ 


$$
\begin{aligned}
& \mathrm{v}_{x}=0, \\
& \mathrm{v}_{y}=-\mathrm{v}_{o}(3 x-1)(x-1),
\end{aligned}
$$

where $\mathrm{v}_{o}$ is the dimensionless liquid speed at $(x, y)=(0,0)$, and is defined using the appropriate slip model, Eq. (A7a) or (A7b).

The mass of liquid within the computational domain must be conserved. For a liquid of constant density, this reduces to the volume constraint,

$$
\int_{0}^{1} h(x) d x=\frac{A}{a^{2}} .
$$

\section{Problem 2}

For model problem 2, the inner region is eliminated and additional boundary conditions are required along $r=R$. The meniscus slope $\theta_{R}$ is applied as a boundary condition on the free surface at $r=R$, replacing the true microscopic dynamic contact angle, $\theta_{D}$. In cylindrical polar coordinates, the velocity boundary conditions applied along the arc, $r=R$, have the general form

$$
\mathbf{v}(r, \phi)=\mathrm{v}_{r}(r, \phi ; \beta) \mathbf{e}_{\mathbf{r}}+\mathrm{v}_{\phi}(r, \phi ; \beta) \mathbf{e}_{\phi},
$$

where $(r, \phi)$ is the polar coordinate system with its origin at the dynamic contact line. The moving solid is at $\phi=0$, and $\phi=\beta(r)$ is the location of the interface. The no-slip boundary condition is applied all along the solid-liquid interface within the computational domain. This truncated domain model allows us to examine how a variety of boundary conditions at $r=R$ affect free surface shapes.

\section{Problem 3}

Model problem 3 consists of a rod entering concentrically into an infinitely deep cylindrical liquid bath. The velocity field is two-dimensional, with no azimuthal component. In the absence of gravity, the outer length scale $a$ $=R_{2}-R_{1}$, where $R_{2}$ and $R_{1}$ are the radii of the outer and inner cylinders, respectively. A new geometric parameter $\delta$ $=R_{1} /\left(R_{2}-R_{1}\right)$ enters the calculations. The set of Eqs. (A1)-(A7) remain unchanged, except for being written in cylindrical co-ordinates. The lower boundary of the computational domain is a hypothetical surface, where we assume a one-dimensional velocity profile given by

$\mathrm{v}_{r}=0$,

$\mathrm{v}_{y}(r)=a_{1} r^{2} / 4+a_{2} \ln r+a_{3}$,

where

$a_{1}=4 \mathrm{v}_{o}\left[2 a_{7} / a_{5}+a_{8}\right] /\left[a_{4} a_{6}+2 a_{7}+a_{5} a_{8}-2 a_{6}(1+\delta)^{2}\right]$,

$a_{2}=\left(\mathrm{v}_{o}-a_{1} a_{5} / 4\right) / a_{6}$,

$a_{3}=-\left[a_{1}(1+\delta)^{2} / 4+a_{2} \ln (1+\delta)\right]$,

$a_{4}=(1+\delta)^{2}+\delta^{2}$,

$a_{5}=(1+\delta)^{2}-\delta^{2}$,

$a_{6}=\ln (1+1 / \delta)$, $a_{7}=\delta^{2} \ln \delta-(1+\delta)^{2} \ln (1+\delta)$,

$a_{8}=1+2 \ln (1+\delta)$.

In order to account for the additional curvature along the azimuthal direction, special " pie-shaped" elements must be chosen for the computations, and the development of the residuals follows the techniques outlined in Bornside. ${ }^{24}$

The volume constraint becomes

$$
2 \pi \int_{\delta}^{1+\delta} h(r) r d r=\frac{V}{a^{3}} .
$$

Here, $V$ is the volume of liquid in the annular space defined by the computational domain.

\section{APPENDIX B: MODULATED WEDGE FLOW SOLUTION}

The modulated-wedge solution developed by $\operatorname{Cox}^{1}$ describes the velocity field in the intermediate region to $\mathrm{O}(1)$ in $\mathrm{Ca}$, as $\mathrm{Ca} \longrightarrow 0$, and is given by

$\mathrm{v}_{r}(r, \phi ; \beta) \sim[-\operatorname{Sin} \beta \operatorname{Cos} \beta(\operatorname{Cos} \phi-\phi \operatorname{Sin} \phi)$

$\left.-\operatorname{Sin}^{2} \beta(\operatorname{Sin} \phi+\phi \operatorname{Cos} \phi)+\beta \operatorname{Cos} \phi\right] / \Delta$,

$\mathrm{v}_{\phi}(r, \phi ; \beta) \sim\left[\phi \operatorname{Cos} \phi \operatorname{Sin} \beta \operatorname{Cos} \beta+\phi \operatorname{Sin} \phi \operatorname{Sin}^{2} \beta\right.$

$-\beta \operatorname{Sin} \phi] / \Delta-\operatorname{Ca}(2 \operatorname{Sin} \beta)[\phi \operatorname{Cos} \phi(\operatorname{Sin} \beta$

$\times \operatorname{Cos} \beta-\beta \operatorname{Cos} 2 \beta)+\phi \operatorname{Sin} \phi\left(\operatorname{Sin}^{2} \beta \operatorname{Cos}^{2} \beta\right.$

$\left.-2 \beta \operatorname{Sin} \beta \operatorname{Cos} \beta+\operatorname{Sin}^{4} \beta+\operatorname{Sin}^{2} \beta\right)$

$+\operatorname{Sin} \phi(\beta \operatorname{Cos} 2 \beta-\operatorname{Sin} \beta \operatorname{Cos} \beta)] / \Delta^{3}$,

where $\Delta=\beta-\sin \beta \cos \beta$ and $\phi=\beta(r)$ represents the free surface.

${ }^{1}$ R. G. Cox, "The dynamics of the spreading of a liquid on a solid surface," J. Fluid Mech. 168, 169 (1986).

${ }^{2}$ V. E. B. Dussan, "On the spreading of liquids on solid surfaces: Static and dynamic contact angles," Annu. Rev. Fluid Mech. 11, 371 (1979).

${ }^{3}$ C. G. Ngan and V. E. B. Dussan, "On the nature of the dynamic contact angle: an experimental study," J. Fluid Mech. 209, 191 (1989).

${ }^{4}$ L. M. Hocking, "A moving fluid interface on a rough surface," J. Fluid Mech. 76, 801 (1976); "A moving fluid interface. Part 2. The removal of the force singularity by a slip flow," 79, 209 (1977); C. Mason Huh, "The steady movement of a liquid in a capillary tube," ibid. 81, 401 (1977).

${ }^{5}$ P. C. Wayner, "Spreading of a liquid film with a finite contact angle by the evaporation/condensation process," Langmuir 9, 294 (1993).

${ }^{6} \mathrm{P}$. Neogi and C. A. Miller, "Spreading kinetics of a drop on a smooth solid surface," J. Colloid Interface Sci. 86, 525 (1982).

${ }^{7}$ D. E. Weidner and L. W. Schwartz, "Contact line motion of shear thinning liquids," Phys. Fluids 6, 3535 (1994).

${ }^{8} \mathrm{~K}$. R. Willson, "The dynamic wetting of polymer melts: an investigation of the role of material properties and the inner scale hydrodynamics," Ph.D. Thesis, Department of Physics, Carnegie Mellon University (1995).

${ }^{9}$ V. E. B. Dussan, "The moving contact line: The slip boundary condition," J. Fluid Mech. 77, 665 (1976).

${ }^{10}$ V. E. B. Dussan, E. Ramé, and S. Garoff, "On identifying the appropriate boundary conditions at a moving contact line: an experimental investigation," J. Fluid Mech. 230, 97 (1991).

${ }^{11}$ J. Marsh, S. Garoff, and V. E. B. Dussan, "Dynamic contact angle and hydrodynamics near a moving contact line," Phys. Rev. Lett. 70, 2778 (1993).

${ }^{12}$ Q. Chen, E. Ramé, and S. Garoff, "'The breakdown of asymptotic hydrodynamic models of liquids spreading at increasing Capillary number," Phys. Fluids 7, 2631 (1995).

${ }^{13}$ E. Ramé and S. Garoff, "Microscopic and macroscopic dynamic interface 
shapes and the interpretation of dynamic contact angles," J. Colloid Interface Sci. 177, 234 (1996).

${ }^{14} \mathrm{O}$. Chen, E. Ramé, and S. Garoff, "The velocity field near moving contact lines," J. Fluid Mech. 337, 49 (1997).

${ }^{15} \mathrm{C}$. Shen and D. Ruth, "Experimental and numerical investigations of the interface profile close to a moving contact line," Phys. Fluids 10, 789 (1998).

${ }^{16} \mathrm{~J}$. Lowndes, "The numerical simulation of the steady movement of a fluid mensicus in a capillary tube," J. Fluid Mech. 101, 631 (1980).

${ }^{17} \mathrm{P}$. Bach and O. Hassager, "An algorith for the use of the Lagrangian specification in Newtonian fluid mechanics and applications to free surface flow,' J. Fluid Mech. 152, 173 (1985).

${ }^{18} \mathrm{~J}$. N. Tilton, "The steady motion of an interface between two viscous liquids in a capillary tube," Ceram. Eng. Sci. Proc. 43, 1371 (1988).

${ }^{19}$ M. Zhou and M. Sheng, "Dynamics of immiscible fluid displacement in a capillary tube," Phys. Rev. Lett. 64, 882 (1990).

${ }^{20} \mathrm{P}$. Sheng and M. Zhou, "Immiscible fluid displacement: Contact line dynamics and the velocity-dependent capillary pressure," Phys. Rev. A 45, 5694 (1992).

${ }^{21}$ D. E. Finlow, P. R. Kota, and A. Bose, "Investigation of wetting hydro- dynamics using numerical simulations,' Phys. Fluids 8, 302 (1996).

${ }^{22}$ I. B. Bazhlekov and A. K. Chesters, "Numerical investigation of the dynamic influence of the contact line region on the macroscopic meniscus shape," J. Fluid Mech. 329, 137 (1996).

${ }^{23}$ R. G. Cox, "Inertial and viscous effects on dynamic contact angles," J. Fluid Mech. 357, 249 (1998).

${ }^{24}$ D. E. Bornside, "Coating flows," Ph.D. Thesis, University of Minnesota (1988)

${ }^{25}$ S. F. Kistler and L. E. Scriven, "Coating flows" in Computational Analysis of Polymer Processing, edited by J. R. A. Pearson and S. M. Richardson (Applied Science, New York, 1983).

${ }^{26}$ P. R. Kota, "Dynamic wetting of solids by surfactant solutions," Ph.D. Thesis, University of Rhode Island (1994); D. E. Finlow, "Numerical simulations of wetting hydrodynamics for pure liquids and surfactant solutions,"' Ph.D. Thesis, University of Rhode Island (1996).

${ }^{27} \mathrm{P}$. Hood, "Frontal solution program for unsymmetric matrices," Int. J. Numer. Methods Eng. 10, 379 (1979).

${ }^{28} \mathrm{H}$. K. Moffatt, "Viscous and resistive eddies near a sharp corner," J. Fluid Mech. 18, 1 (1964). 